\section{Les nouvelles promesses de la metformine}

> La metformine est l'antidiabétique oral de première intention lors de la prise en charge thérapeutique du diabète de type 2. Son mécanisme d'action est resté longtemps imprécis et commence seulement à être dévoilé. Des données épidémiologiques récentes ont montré que cet antidiabétique exerce également une protection cardiovasculaire et présente des propriétés antitumorales, indépendamment de son action sur la glycémie. Nous présentons dans cette revue les différents mécanismes d'action de la metformine dans ses effets bénéfiques sur la glycémie, les pathologies cardiovasculaires et le cancer. <

\section{Histoire passionnante de la metformine}

L'histoire de la metformine remonte au Moyen Âge en Europe avec l'utilisation du galéga officinal (Galega officinalis) (Figure 1), nommé aussi «lilas français » ou «rue-des-chèvres». Cette plante médicinale est utilisée pour soigner, entre autres, les manifestations du diabète sucré chez l'homme et pour augmenter la production de lait (propriété galactogène) chez le bétail [1]. Dès le XIX ${ }^{e}$ siècle, les fleurs et les graines du galéga sont utilisées spécifiquement pour leurs effets antihyperglycémiants. Les principes actifs hypoglycémiants de la plante sont la guanidine et l'isoamylène guanidine (galégine) (Figure l) isolée par le pharmacien français Georges Tanret en 1914. Bien que la galégine ait été utilisée avec succès, elle fut rapidement délaissée à cause de sa toxicité. Ensuite, les diguanidines, molécules contenant deux guanidines reliées par une chaîne alkyl de longueur variable, sont produites dans les années 1920. Deux diguanidines, les synthalines $A$ et $B$ (décaméthylène diguanidine et dodécaméthylène diguanidine) (Figure 1), ont été utilisées cliniquement, mais elles seront abandonnées rapidement car leur effet thérapeutique n'a pas pu être dissocié de leur toxicité. Les biguanides, composés issus de la condensation de deux molécules de guanidine

\section{Vers une meilleure compréhension de ses mécanismes d'action}

Marc Foretz $^{1,2,3}$, Benoit Viollet ${ }^{1,2,3}$

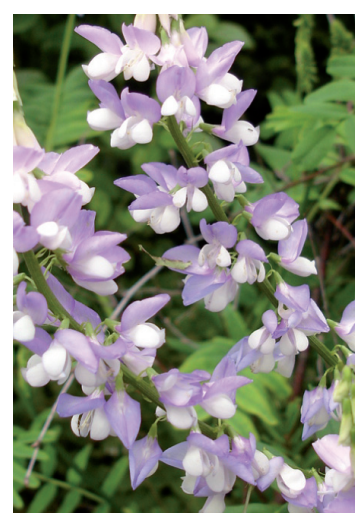

${ }^{1}$ Inserm U1016, Institut Cochin, département d'endocrinologie, métabolisme et diabète, 24, rue du Faubourg Saint Jacques, 75014 Paris, France ; ${ }^{2}$ CNRS, UMR8104, Paris, France ; ${ }^{3}$ Université Paris Descartes, Sorbonne Paris Cité, France. marc.foretz@inserm.fr benoit.viollet@inserm.fr avec élimination d'une molécule d'ammoniac, sont synthétisées à la même époque. La metformine ( $\mathrm{N}, \mathrm{N}$-diméthylbiguanide) (Figure 1) est produite pour la première fois en 1922 à Dublin par Werner et Bell. En 1929, ses propriétés hypoglycémiantes sont mises en évidence par deux équipes allemandes [1]. Toutefois, ces découvertes sont éclipsées par la découverte de l'insuline en 1921, et il faudra attendre la fin des années 1950 pour redécouvrir le potentiel clinique des biguanides dans le traitement du diabète.

En 1957, Jean Sterne, un médecin français, réalisa les premiers essais cliniques chez l'homme de la metformine utilisée comme agent antidiabétique oral. Il démontra que, de toutes les autres biguanides testées, la metformine possèdait le meilleur rapport bénéfice/risque [2]. À la suite de ces travaux, la metformine fut commercialisée pour la première fois en France sous le nom évocateur de Glucophage par les laboratoires Aron en 1959. En 1958, la phenformine (phényléthylbiguanide) et la buformine (monobutylbiguanide) (Figure 1) sont privilégiées comme agents antidiabétiques, respectivement aux États-Unis et en Allemagne. Ces deux biguanides étaient plus actives que la metformine, mais, à la suite de plusieurs cas d'acidose lactique mortels et d'accidents cardiaques, la phenformine a été retirée du marché américain en 1976. Après confirmation d'une faible incidence de cas d'acidose lactique, la metformine a continué à être prescrite très largement en Europe. Les accidents liés à la prise de phenformine ont toutefois entaché la réputation de la metformine et empêché sa diffusion aux États-Unis. L'étude multicentrique britannique UKPDS (United Kingdom prospective diabetes study) permettra de confirmer la place de référence de la metformine dans le traitement du diabète 


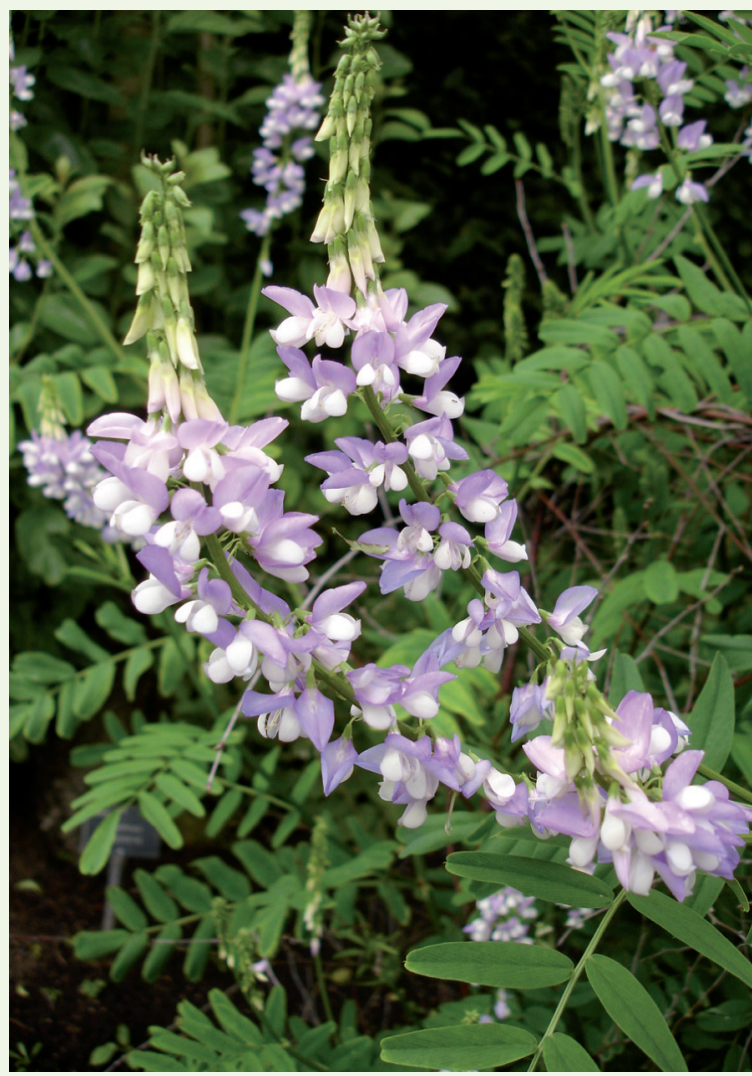

\begin{tabular}{|c|c|}
\hline Guanidine & $\begin{array}{r}\mathrm{NH} \\
\mathrm{NH}_{2}-\mathrm{C}-\mathrm{NH}_{2}\end{array}$ \\
\hline Galégine & $\begin{array}{l}\mathrm{CH}_{3} \backslash \mathrm{C}=\mathrm{CH}-\mathrm{CH}_{2}-\mathrm{NH}-\mathrm{C}-\mathrm{NH}_{2} \\
\mathrm{CH}_{3},\end{array}$ \\
\hline Synthaline A & $\mathrm{NH}_{2}-\mathrm{NH}$ \\
\hline Synthaline B & 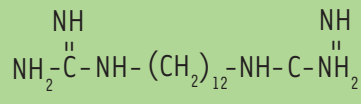 \\
\hline Biguanide & $\underset{\mathrm{NH}}{\mathrm{NH}}-\stackrel{\mathrm{CH}}{\mathrm{C}}-\mathrm{NH}-\mathrm{C}-\mathrm{NH}_{2}$ \\
\hline Metformine & $\begin{array}{l}\mathrm{CH}_{3} \backslash \mathrm{NH} \\
\mathrm{CH}_{3} / \mathrm{N}-\mathrm{C}-\mathrm{NH}-\mathrm{C}-\stackrel{\mathrm{NH}}{\mathrm{NH}}\end{array}$ \\
\hline Phenformine & 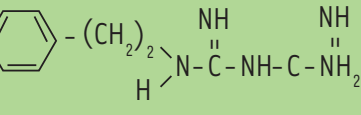 \\
\hline Buformine & 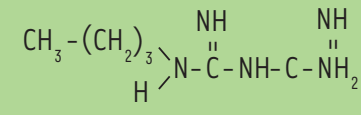 \\
\hline
\end{tabular}

Figure 1. Origine, historique et structure chimique de la metformine et des molécules apparentées. Le galéga officinal est une plante médicinale utilisée dès le Moyen Âge pour soigner les symptômes du diabète sucré. Au début du xxe siècle, la galégine, un alcaloïde appartenant à la famille des guanidines, est isolée comme principe actif du galega. La galégine possède des propriétés antidiabétiques, mais se révéla rapidement toxique. Les synthalines A et B, qui contiennent deux guanidines reliées par une chaîne alkyl, ont été utilisées cliniquement dans les années 1920 , mais également abandonnées en raison de leur toxicité. Ensuite, les biguanides, qui résultent de la condensation de deux guanidines, sont produites. La metformine est décrite en 1929 et testée comme antidiabétique chez l'homme en 1957 par le français Jean Sterne. Deux autres biguanides, la phenformine et la buformine, sont utilisées à la même époque, mais à cause d'un risque d'acidose fatale important, elles ont été retirées du marché. Unique représentante de la classe des biguanides, la metformine est aujourd'hui l'antidiabétique oral de référence pour traiter le diabète de type 2. Photographie: Wikimedia commons, Epibase.

de type 2 [3]. Initialement réticents envers ce médicament « européen », les États-Unis l'adoptent seulement en 1995 et il fait maintenant partie dans ce pays des 10 médicaments les plus utilisés. Depuis 2002, la metformine est disponible sous forme générique. Aujourd’hui, elle est prescrite à plus de 120 millions de personnes dans le monde, et est ainsi devenue l'antidiabétique le plus utilisé.

\section{Effet antidiabétique de la metformine}

La metformine est utilisée avec succès depuis plus d'un demi-siècle dans le traitement du diabète de type $2^{1}$. Grâce à son efficacité et ses effets secondaires limités, la metformine est recommandée, en l'absence de contre-indications, comme l'antidiabétique oral de première intention

${ }^{1}$ Voir le numéro thématique de médecine/sciences consacré au diabète (Diabète : approches thérapeutiques émergentes, $\mathrm{m} / \mathrm{s} \mathrm{n}^{\circ} 8-9$, vol. 29, août-septembre 2013). pour traiter les patients diabétiques de type 2 par l'American diabetes association et l'European association of the study of diabetes. La metformine réduit l'hyperglycémie sans risque d'hypoglycémie contrairement à d'autres antidiabétiques comme les sulfonylurées et l'insuline. Pour cette raison, elle est considérée comme un agent antihyperglycémique. La metformine améliore également la sensibilité à l'insuline, entraînant une réduction de la résistance à l'insuline et une diminution des concentrations plasmatiques de cette hormone.

Les effets secondaires liés à la prise de metformine sont principalement des troubles gastro-intestinaux incluant des douleurs abdominales, des nausées, des vomissements et des diarrhées. Ces symptômes surviennent au début du traitement, mais disparaissent en général rapidement. L'acidose lactique est le risque majeur du 


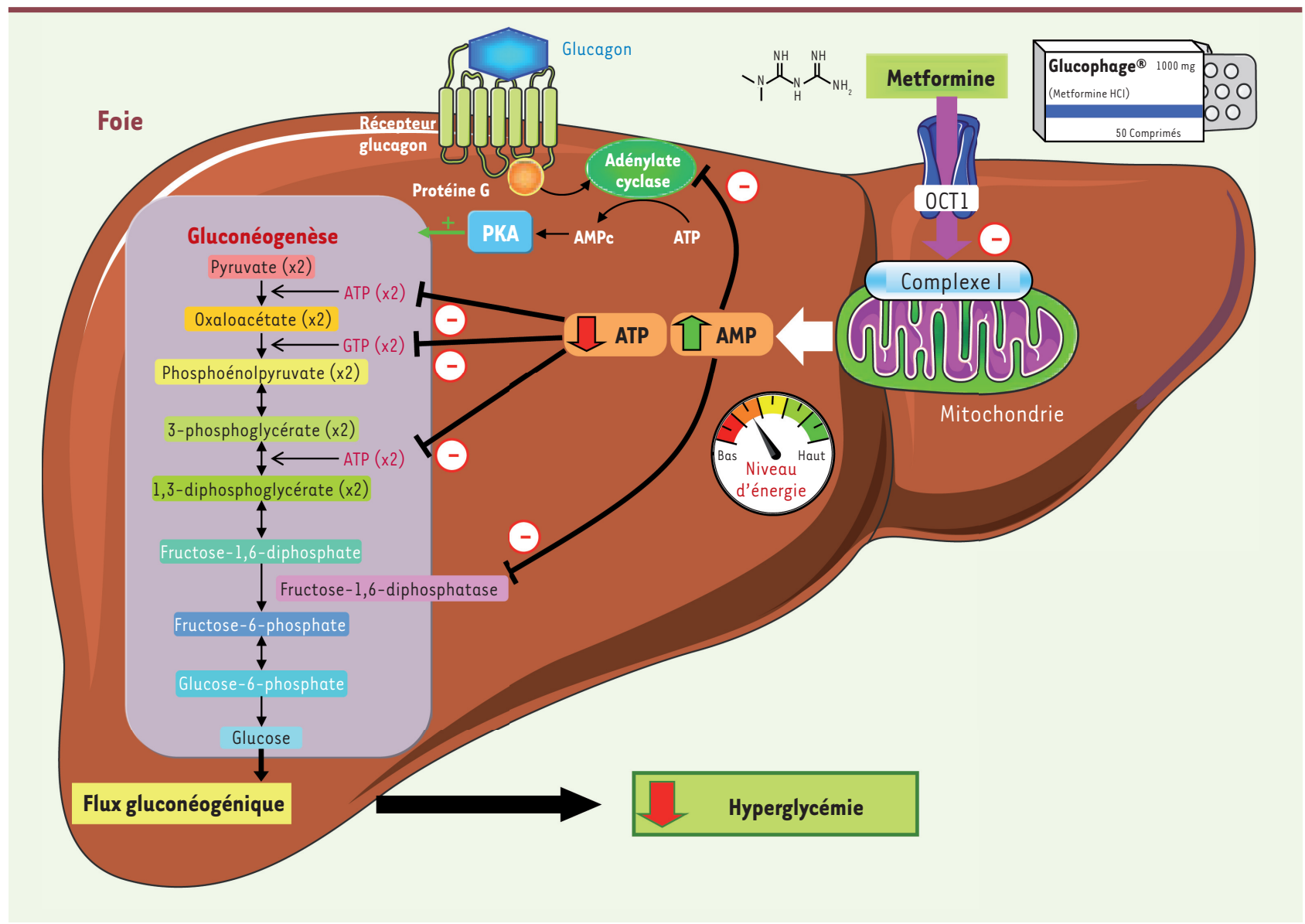

Figure 2. Mécanismes d'inhibition de la production de glucose par la metformine via la diminution du potentiel énergétique dans le foie. La metformine est transportée dans les hépatocytes par le transporteur OCTl. Dans la cellule, elle inhibe partiellement la chaîne respiratoire mitochondriale au niveau du complexe I, qui est sa cible primaire. II en résulte une diminution du niveau d'énergie dans la cellule qui se traduit par une baisse des concentrations intracellulaires d'ATP et une augmentation concomitante des concentrations d'AMP. La gluconéogenèse est une voie métabolique énergivore qui nécessite 4 ATP et 2 GTP par molécule de glucose produite. La diminution d’ATP en réponse à la metformine réduit en conséquence la production de glucose. De plus, l'accumulation de l'AMP inhibe de manière allostérique la fructose-1,6-diphosphatase, une enzyme clé de la gluconéogenèse, et diminue l'activation de l'adénylate cyclase stimulée par le glucagon. II en résulte une diminution du flux gluconéogénique et une amélioration de l'hyperglycémie chez le patient diabétique de type 2.

traitement par les biguanides. Elle résulte d'une augmentation de la glycolyse anaérobie qui entraîne une accumulation de lactate dans la circulation provoquant une diminution du $\mathrm{pH}$ sanguin à l'origine d'un état de choc. Elle fut observée avec la phenformine, mais elle est exceptionnelle avec la metformine. Son incidence est estimée à 3 cas pour 100 000 patients par an, soit un risque 10 à 20 fois moins important qu'avec la phenformine. À cause du risque d'acidose lactique, la metformine est contre-indiquée dans toutes les situations pathologiques pouvant provoquer, soit une hypoxie tissulaire ou une ischémie (comme en cas d'insuffisance cardiaque ou pulmonaire), soit une accumulation de metformine dans l'organisme par défaut d'élimination (comme en cas d'insuffisance rénale ou hépatique). Toutefois, le rôle causal de la metformine dans la plupart des cas d'acidoses lactiques rapportés n'est pas totalement avéré [4]. La metformine n'expose pas au risque d'hypoglycémie par interaction médicamenteuse. Ainsi, lorsque son utilisation en monothérapie est insuffisante, elle peut être prescrite en combinaison avec d'autres antidiabétiques, en absence de contre-indications.

À l'inverse des autres antidiabétiques, la metformine n'entraîne pas de gain de poids. Au contraire, une perte pondérale est souvent observée chez les patients obèses [3]. Cet effet sur le poids pourrait être une conséquence de la diminution de l'insulinémie, bien qu'un effet anorexigène propre ait été suggéré [5]. Elle a également une action bénéfique sur le profil des lipides circulants en diminuant les concentrations plasmatiques de triglycérides et de cholestérol, procurant ainsi une protection anti-athérogène [3]. Des études chez l'homme et chez la souris suggèrent que la metformine améliore la stéatose hépatique $[6,7]$. La stéatose 
hépatique non alcoolique est une pathologie fréquemment associée au diabète de type 2 ; elle résulte d'une accumulation de triglycérides dans le foie. La diminution du contenu lipidique des hépatocytes en réponse à la metformine limiterait le phénomène de lipotoxicité et contribuerait à améliorer la sensibilité à l'insuline dans le foie $[8,9]$. Par ailleurs, la metformine prévient l'apparition du diabète de type 2 chez des sujets à risque, en particulier chez les patients en surpoids [10], et améliore de manière efficace et sûre le diabète gestationnel [11]. Le syndrome des ovaires polykystiques est un trouble endocrinien caractérisé par une absence d'ovulation chronique associée à une résistance à l'insuline. L'amélioration de la résistance à l'insuline par la metformine chez ces femmes est concomitante d'un rétablissement du cycle menstruel et d'une augmentation significative de la fertilité [12]. Par son efficacité, sa sureté et ses multiples bienfaits, la metformine est devenue le gold standard en matière d'antidiabétique. De nos jours, le développement d'un médicament est orienté pour agir sur une cible pharmacologique préalablement identifiée. Découverte à une époque antérieure aux critères de conception actuels d'un médicament, la metformine a été utilisée pendant plusieurs décennies sans que les détails moléculaires de son action soient connus. Depuis une dizaine d'années, la metformine est devenue l'objet d'intenses recherches, et ses mécanismes d'action sont de mieux en mieux compris. Dans les paragraphes suivants, nous ferons le point des découvertes récentes concernant ces mécanismes et des nouvelles utilisations potentielles de la metformine.

\section{Le foie est l'organe cible de la metformine}

Le foie occupe une place centrale dans l'action antihyperglycémique de la metformine. En effet, celle-ci réduit l'hyperglycémie en diminuant la production de glucose hépatique par inhibition de la gluconéogenèse, qui est anormalement augmentée chez les patients diabétiques de type 2. Dans une moindre mesure, elle diminue l'absorption gastro-intestinale du glucose et augmente son utilisation dans le muscle. À long terme, la metformine augmente également la sensibilité à l'insuline dans le foie.

Après administration orale, la metformine est absorbée par l'intestin, et ses concentrations dans la veine porte sont importantes. II en résulte que le foie est exposé à des concentrations de metformine plus élevées que les autres tissus périphériques. Contrairement à la phenformine, la metformine est une molécule très hydrophile et son passage à travers la membrane plasmique par diffusion passive est pratiquement nul. Les transporteurs de cations organiques $0 \mathrm{CTl}$, -2 et -3 (organic cation transporter 1,2 et 3 ) sont les principaux transporteurs de la metformine. Le transporteur $\mathrm{OCTl}$ est fortement exprimé dans l'intestin, le foie et le rein, et faiblement dans les autres tissus. Ainsi, les transporteurs OCT participent à l'absorption et à l'excrétion de la metformine, respectivement dans l'intestin et le rein. L'expression importante du transporteur OCTl dans le foie entraîne une accumulation de la metformine plus importante dans cet organe que dans les autres tissus. Cette accumulation hépatique est accentuée par le fait que la metformine n'est pas métabolisée par le foie, et est excrétée par la voie urinaire sans modification. Ces caractéristiques pharmacocinétiques positionnent le foie comme l'organe cible de la metformine. Ainsi, la délétion du gène 0 ctl dans le foie chez la souris entraîne une diminution importante de l'accumulation de metformine au niveau hépatique. De même chez l'homme, l'existence de polymorphismes au niveau du gène $0 C T 1$ réduit l'action de la metformine pour abaisser l'hyperglycémie [13]. La metformine est également prise en charge par le transporteur MATE (multidrug and toxin extrusion) qui contribue à son excrétion rénale, et par le transporteur PMAT (plasma membrane monoamine transporter) qui participe à son absorption intestinale.

\section{Mécanismes antihyperglycémiques de la metformine : une question d'énergie}

La découverte la plus significative dans la compréhension du mécanisme d'action cellulaire de la metformine a été réalisée par l'équipe de Xavier Leverve à Grenoble (France). En 1993, cette équipe avait déjà montré que l'inhibition de la production de glucose par la metformine dans des hépatocytes était associée à une diminution des concentrations intracellulaires d'ATP [14]. En 2000, ce groupe a montré pour la première fois que la metformine inhibe modérément le complexe I (NADH:ubiquinone oxydoréductase) de la chaîne respiratoire mitochondriale de manière spécifique et non toxique, avec pour conséquence une diminution de I'ATP produit par phosphorylation oxydative, et une augmentation modérée du rapport AMP/ATP dans les hépatocytes [15]. Ainsi, le complexe I de la chaîne respiratoire est la cible primaire de la metformine.

Le mécanisme précis par lequel la metformine module l'activité du complexe I de la chaîne respiratoire n'est pas clairement établi. Contrairement à la roténone, un autre inhibiteur du complexe I, la metformine est incapable d'inhiber la respiration dans des mitochondries isolées ou dans des cellules perméabilisées ; cette inhibition est uniquement observée dans des cellules intactes ou des mitochondries isolées provenant de cellules prétraitées par la metformine [15]. Il est important de souligner que cette inhibition est partielle et n'excède pas $40 \%$ de l'activité maximale du complexe I (contre $80 \%$ pour la roténone), suggérant que la metformine s'accumule dans la matrice mitochondriale de façon auto-limitante. En effet, comme la metformine est chargée positivement et très hydrophile, elle entre dans la mitochondrie grâce au potentiel de membrane. L'augmentation de sa concentration dans la matrice inhibe progressivement la chaîne respiratoire. Ceci va conduire à une baisse du potentiel membranaire et empêcher ainsi son accumulation dans la mitochondrie [16]. Ce mécanisme d'auto-contrôle 


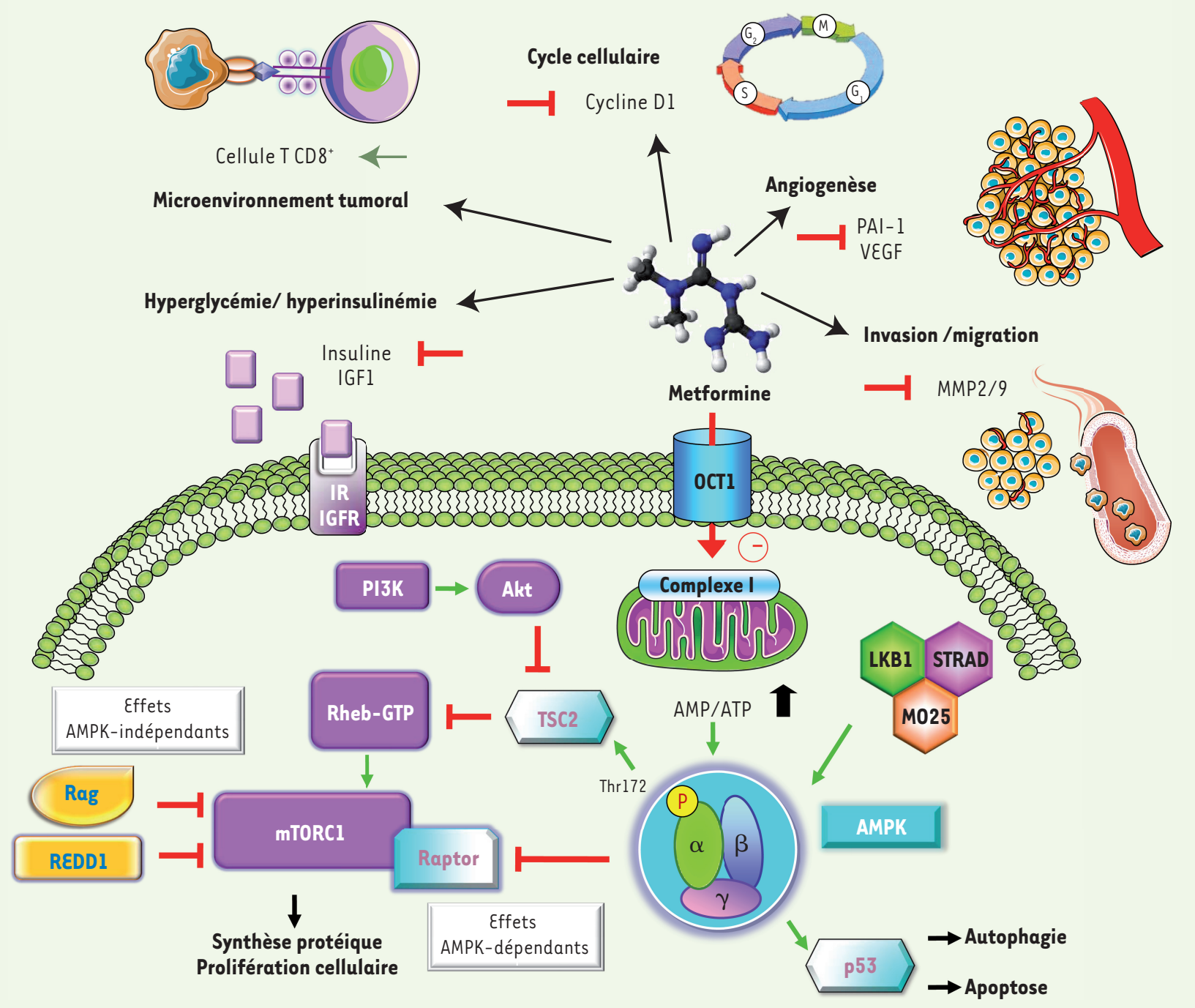

Figure 3. Principaux mécanismes d'action décrits pour l'inhibition de la croissance tumorale par la metformine. Plusieurs modes d'actions ont été proposés en raison de la diversité des modèles de cancer étudiés in vivo et in vitro. Au niveau systémique, la metformine réduit les taux circulants d'insuline et d'IGFl, permettant de bloquer l'induction des voies de signalisation PI3K/AKT/mTORCl et la prolifération cellulaire. Au niveau cellulaire, la metformine inhibe la voie mTORCl (mammalian target of rapamycin complex 1) par des mécanismes dépendants d'une activation de I'AMPK par la phosphorylation de TSC2 (tuberous sclerosis complex 2) et de raptor (regulatory associated protein of mTOR). Cependant, la metformine est capable d'inhiber la voie mTORCl par des mécanismes indépendants de l'AMPK, en inhibant les GTPases Rag et en induisant l'expression de REDDl. Des actions au niveau de la régulation du cycle cellulaire par l'inhibition de l'expression de la cycline Dl sont possibles. Au niveau du microenvironnement tumoral, l'induction de la génération de cellules T mémoire contribuent également aux effets bénéfiques de la metformine. La metformine provoque une réduction de l'angiogenèse des cellules néoplasiques par une baisse des taux circulants de PAl-1 (plasminogen activator inhibitor-1) et de VEGF (vascular endothelial growth factor). Une inhibition de la formation de métastases par une réduction de l'activité des métalloprotéinases MMP2 et -9 a été décrite.

expliquerait le faible risque d'acidose lactique observé avec la metformine, alors que ce risque est fortement accru avec la phenformine qui, elle, s'accumule dans la membrane mitochondriale à cause de sa chaîne latérale hydrophobe (Figure 1). La metformine est un très bon chélateur d'ions divalents. Récemment, une étude a suggéré que l'effet de la metformine résulterait de ses propriétés à interagir avec le cuivre, sans toutefois en expliquer les conséquences dans la cellule [17].
De manière secondaire, l'inhibition du complexe I de la chaîne respiratoire entraîne dans la cellule une diminution du statut énergétique et modifie les flux métaboliques, ainsi que l'activité d'enzymes régulées par l'ATP, I'ADP et I'AMP. En particulier, Zhou et al. ont démontré en 2001 que l'AMPK (AMP-activated protein kinase) est activée par la metformine [18]. L'AMPK est une sérine 
thréonine kinase extrêmement conservée au cours de l'évolution [19]. Cette protéine kinase joue le rôle de senseur métabolique sensible aux variations d'énergie intracellulaire, son activation permettant d'ajuster en permanence la production et l'utilisation d'énergie dans la cellule. L'AMPK est un hétérotrimère composé d'une sous-unité catalytique $\alpha$ et de deux sous-unités régulatrices $\beta$ et $\gamma$ [19]. Elle est activée de manière allostérique par l'AMP et par la phosphorylation de la thréonine 172 de la sous-unité catalytique $\alpha$ par une AMPK kinase, en particulier le suppresseur de tumeur LKBI (liver kinase B1). L'AMPK est activée par une diminution du rapport intracellulaire ATP/AMP suite à un stress métabolique comme l'exercice, le jeûne ou l'hypoxie. Une fois activée, elle inhibe les voies métaboliques anaboliques consommatrices d'ATP et active les voies cataboliques productrices d'ATP afin de rétablir le rapport ATP/ AMP intracellulaire. Cette régulation implique la phosphorylation par I'AMPK d'enzymes métaboliques clés et de facteurs de transcription pour aboutir au final à une modification du métabolisme du glucose et des lipides, de la synthèse protéique et de la croissance cellulaire [20].

Plusieurs mécanismes moléculaires ont été proposés pour expliquer les effets hépatiques de la metformine dans l'inhibition de la gluconéogenèse, incluant l'amélioration des effets de l'insuline, des changements d'activité d'enzymes clés ou encore une diminution du transport hépatique des substrats gluconéoformateurs. L'hypothèse d'une inhibition transcriptionnelle des gènes intervenant dans la gluconéogenèse par activation de l'axe LKBI/AMPK en réponse à la metformine a prévalu pendant plusieurs années $[8,19,21]$. Toutefois, ce mécanisme a récemment été invalidé par des expériences réalisées chez des souris dont les gènes $L k b 1$ ou Ampk avaient été inactivés dans le foie [9, 22]. Ces travaux ont clairement montré que l'inhibition, à court terme, de la production de glucose hépatique par la metformine est indépendante de la voie LKBI/AMPK et d'un effet sur l'expression des gènes. De plus, cette étude a démontré que la diminution du statut énergétique hépatique constitue le facteur critique dans l'inhibition de la production de glucose hépatique par la metformine (Figure 2). En effet, la gluconéogenèse est une voie métabolique coûteuse en énergie puisque six équivalents ATP (4 ATP et 2 GTP) sont nécessaires pour former une molécule de glucose. En conséquence, la diminution des concentrations intracellulaires en ATP par l'inhibition du complexe I mitochondrial réduit le flux gluconéogénique. De plus, l'augmentation des concentrations intracellulaires d'AMP inhibe de manière allostérique la fructose-1,6-biphosphatase, une enzyme clé de la gluconéogenèse, contribuant à l'inhibition de la production de glucose (Figure 2). Récemment, il a été suggéré que l'augmentation de l'AMP intracellulaire induite par la metformine réduit la gluconéogenèse stimulée par le glucagon. En effet, l'AMP inhibe l'activité de l'adénylate cyclase stimulée par le glucagon en se liant sur un site inhibiteur appelé site $P$ au niveau de l'enzyme. Cette inhibition diminue les concentrations intracellulaires d'AMP cyclique (AMPc) et l'activité de la PKA (protéine kinase $A$ ), conduisant à une réduction de la production de glucose stimulée par le glucagon [23] (Figure 2).

Ainsi, la metformine diminue l'hyperglycémie par une inhibition de la production de glucose hépatique en réduisant le flux gluconéogénique par une action purement énergétique. Ce mécanisme implique une dimi- nution de l'ATP et une augmentation concomitante de I'AMP via l'inhibition du complexe I de la chaîne respiratoire, cet effet étant indépendant de la voie LKBI/AMPK.

\section{Effet cardioprotecteur : la botte secrète de la metformine}

Les complications cardiovasculaires demeurent la principale cause de décès chez les patients atteints de diabète de type 2. Dans l'étude longitudinale UKPDS [3], il a été constaté que le traitement par la metformine réduit de manière significative la mortalité toutes causes confondues. Contrairement à d'autres agents antidiabétiques (insuline ou sulfonylurés), la metformine entraîne une diminution d'environ $40 \%$ de la mortalité liée aux pathologies cardiovasculaires, comme l'infarctus du myocarde ou les attaques cérébrales, indiquant que la protection cardiovasculaire de la metformine pourrait être dissociée de son action antihyperglycémique. Des travaux chez le rat non diabétique ont confirmé que la metformine améliore la fonction cardiaque et réduit la taille des lésions après l'induction d'un infarctus du myocarde [24]. Cet effet bénéfique résulte d'un phénomène de préconditionnement myocardique induit de manière plus ou moins permanente par la metformine. Un effet fondamental de la metformine est d'induire l'adaptation métabolique du myocarde lors des situations énergétiques critiques, comme l'ischémie. Dans un modèle d'insuffisance cardiaque chez le rongeur, la metformine favorise l'adaptation métabolique du cœur en stimulant l'utilisation progressive du glucose à la place des acides gras. Cette action serait dépendante d'une activation de l'AMPK qui participe à la stimulation de la capture du glucose et du flux glycolytique face à la privation d'oxygène [25].

L'utilisation de la metformine chez les patients diabétiques ayant des antécédents d'insuffisance cardiaque a montré des effets bénéfiques avec une réduction de la mortalité et de la morbidité [26]. Un des mécanismes proposé pour le développement de la cardiomyopathie diabétique et l'insuffisance cardiaque est un défaut de l'autophagie dans le cœur. La metformine permet de restaurer l'autophagie chez des souris diabétiques et d'empêcher l'apparition de lésions cardiaques. Une activation de l'AMPK est nécessaire, puisque la metformine est inefficace chez les souris diabétiques présentant un défaut d'activité AMPK dans le cœur [27].

L'origine des effets cardioprotecteurs de la metformine est multiple et implique également des actions affectant les systèmes macro- et microvasculaires. La metformine est connue pour exercer un effet protecteur sur l'endothélium vasculaire à la fois par une diminution de la production de radicaux libres et une réduction de la 


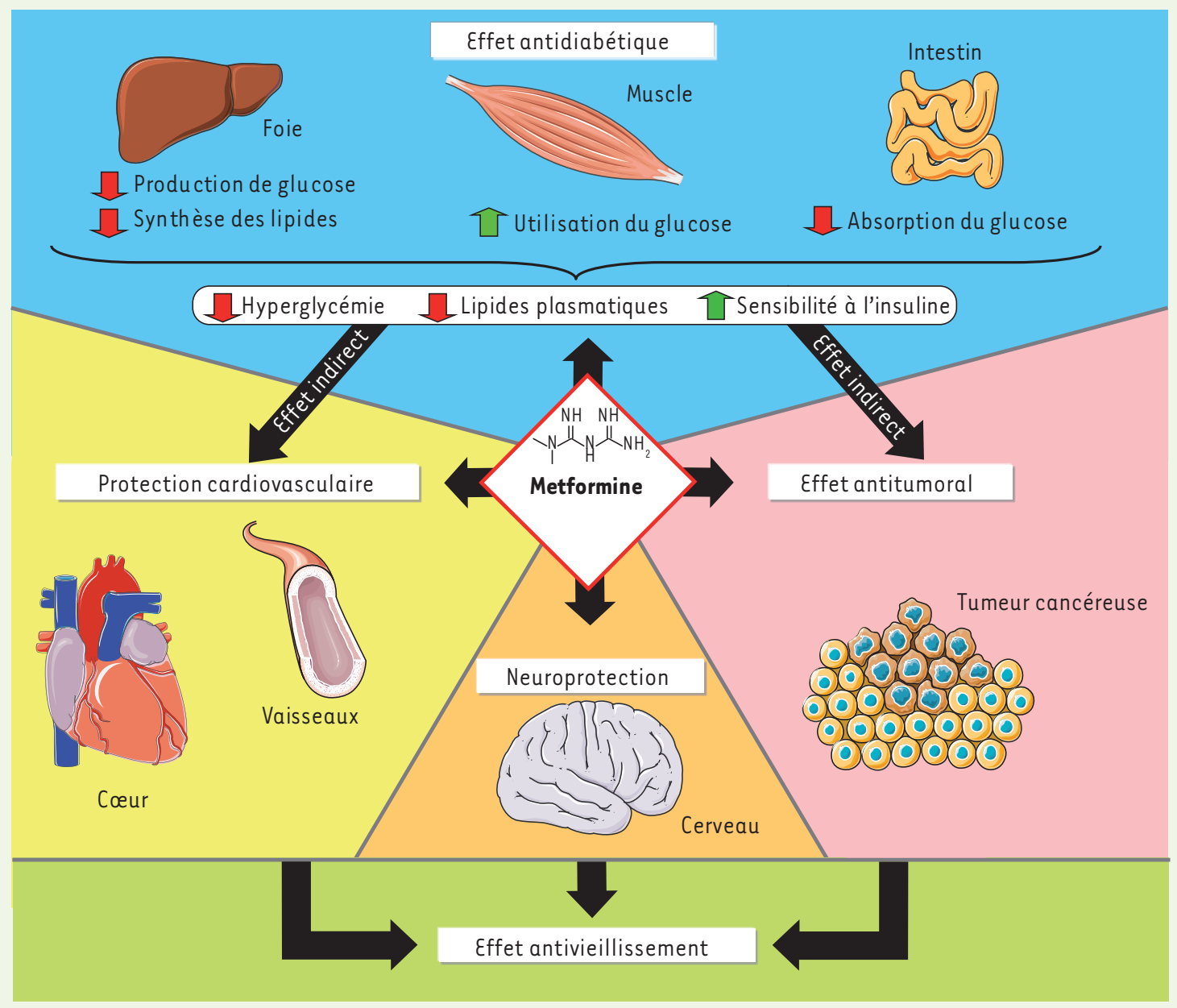

Figure 4. Résumé des principaux effets de la metformine. La metformine exerce ses effets antidiabétiques principalement au niveau du foie par l'inhibition de la gluconéogenèse et, dans une moindre mesure, au niveau de l'intestin et du muscle, pour aboutir à une diminution de l'hyperglycémie et des lipides circulants, ainsi qu'à une augmentation de la sensibilité à l'insuline. Ces améliorations procurent de manière indirecte une protection cardiovasculaire et un effet antitumoral. De plus, la metformine réduit de manière directe le risque de maladies cardiovasculaires par des actions affectant à la fois les systèmes macro- et micro-vasculaires, et produit une action antitumorale directement sur les cellules cancéreuses. Par ailleurs, la metformine aurait un effet neuroprotecteur dans les pathologies neurodégénératives. Les effets bénéfiques de la metformine s'apparentent à ceux observés au cours de la restriction calorique, lesquels concourent à prolonger l'espérance de vie. Ainsi, la metformine peut être considérée comme un mimétique de la restriction calorique qui pourrait avoir des propriétés antivieillissement.

formation de protéines glyquées, qui sont à l'origine d'un stress oxydant cellulaire et d'une inflammation. En raison des effets vasculaires et des propriétés anti-athérogène de la metformine, son utilisation pourrait être étendue aux patients non diabétiques présentant un risque de développer des pathologies vasculaires.

\section{Effet antitumoral : nouvelle promesse de la metformine}

Les premières études qui suggéraient une utilisation possible de la metformine en cancérologie ont été publiées à partir de 2005 [28]. Ensuite, de nombreuses autres études épidémiologiques observationnelles ont confirmé cette réduction du risque de développer un cancer ou de décéder d'un cancer chez les patients diabétiques de type 2 traités depuis plusieurs années par la metformine [29]. De plus, la mise en évidence d'une relation entre la dose de metformine ou la durée du traitement et la protection observée a renforcé la possibilité d'un effet antitumoral de la metformine [28]. Ces études s'accordent également quant à l'efficacité de la metformine sur la réduction de l'incidence du cancer et de la mortalité associée pour tous les types de cancer pris globalement, même si certaines études ont révélé des effets spécifiques pour les cancers digestifs et du sein [29]. Un grand nombre de mécanismes ont été décrits 
pour expliquer les effets bénéfiques de la metformine (Figure 3), car ils dépendent des caractéristiques métaboliques et moléculaires de chaque tumeur.

Effet de la metformine sur les taux circulants d'insuline et d'IGF1 Plusieurs études épidémiologiques ont rapporté une forte association entre le diabète de type 2 et le risque élevé de certains cancers, en particulier du côlon, de l'endomètre, du rectum et du sein, par rapport à leur incidence dans la population non diabétique. L'hyperinsulinémie compensatrice et l'hyperglycémie chronique, caractéristiques du diabète de type 2 , seraient à l'origine de ce lien entre diabète et cancer. $\varepsilon n$ effet, l'insuline est connue pour favoriser la prolifération cellulaire et augmenter les taux circulants d'IGFl (insulin-like growth factor 1), qui agit comme un facteur de croissance dans le développement tumoral. Il est également bien établi que le glucose est utilisé comme substrat énergétique préférentiel par les cellules en phase de prolifération, et l'hyperglycémie pourrait contribuer à améliorer la croissance et la survie des cellules cancéreuses. Ainsi, un certain nombre de traitements visant à réduire l'hyperglycémie et/ou l'hyperinsulinémie ont permis de réduire le risque de cancer. L'inhibition de la progression tumorale par la metformine a été validée par l'analyse de nombreux modèles murins de carcinogenèse, en accord avec les études épidémiologiques observationnelles. Pour certains modèles, l'inhibition de la prolifération tumorale a été directement liée à la réduction des taux circulants d'insuline et d'IGFl par la metformine (Figure 3) et à celle de l'activité des récepteurs à activité tyrosine kinase [30], mais cet effet n'a pas été retrouvé dans d'autres modèles, indiquant un mode d'action indépendant d'une baisse de l'insulinémie.

\section{Effet antiprolifératif de la metformine}

Plusieurs études cliniques ont indiqué que la metformine est capable de conférer un effet protecteur contre le cancer indépendamment de la présence d'un diabète [31]. Ce résultat a été conforté par de nombreuses évidences obtenues in vitro montrant que la metformine inhibe directement la prolifération des cellules cancéreuses [32, 33]. II est important de noter que la sensibilité des cellules tumorales à la metformine est variable, et l'effet antiprolifératif n'est observé que pour de fortes concentrations de metformine (5-50 mM), bien supérieures à celles obtenues pour un usage thérapeutique chez le diabétique (concentrations plasmatiques de l'ordre de 10 à $40 \mu \mathrm{M}$ ). Néanmoins, la metformine s'accumule dans certains organes comme l'intestin ou le foie, permettant d'atteindre des concentrations intracellulaires beaucoup plus élevées qu'au niveau plasmatique.

\section{Régulation de l'axe AMPK/ mTOR}

Plusieurs études ont suggéré que les effets antiprolifératifs de la metformine pourraient dépendre d'une activation de I'AMPK, suite à l'inhibition du complexe I de la chaîne respiratoire. En accord avec cette hypothèse, l'inhibition de l'AMPK ou l'utilisation de cellules déficientes en LKBI bloque l'effet antiprolifératif de la metformine [32]. Par ailleurs, l'activation de l'AMPK par un activateur direct (A-769662) permet de réduire la progression tumorale de manière identique à celle qu'entraîne la metformine dans un modèle de carcinogenèse induit par la perte du suppresseur de tumeur PTEN (phosphatase and tensin homolog), renforçant le rôle de l'AMPK dans le contrôle de la prolifération cellulaire [34]. L'AMPK inhibe la voie de signalisation mTORCl (mammalian target of rapamycin complex 1 ) qui intègre, via les voies de signalisation Ras/દRK (extracellular signal-regulated kinase) et PI3K (phosphoinositide 3-kinase)/AKT (protein kinase B), les signaux nutritionnels et mitogènes pour contrôler la croissance et la prolifération cellulaires [53]. L'AMPK phosphoryle directement le suppresseur de tumeur TSC2 (tuberous sclerosis complex 2) et la protéine régulatrice raptor (regulatory associated protein of mTOR) pour induire une suppression rapide de l'activité de la voie mTORCl (Figure 3). Bien que la voie LKBl/AMPK soit décrite comme un acteur majeur dans l'inhibition de la voie mTORCl par la metformine, certains mécanismes indépendants de I'AMPK et impliquant les GTPases Rag ou la protéine REDDl (regulated in development and DNA damage response 1) ont été proposés [35] (Figure 3). Cette inhibition de la voie mTORCl par la metformine conduit à la diminution de l'expression du récepteur de l'EGF (epidermal growth factor) et de sa forme oncogénique HER2 (erbB-2) dans des lignées de cancer du sein.

\section{Régulation du cycle cellulaire}

Une analyse des profils d'expression génique dans des tumeurs de cancer du sein traitées par la metformine a révélé une réduction de l'expression de nombreux gènes intervenant au cours de la mitose. $\varepsilon$ n accord avec ces résultats, le traitement de cellules cancéreuses du poumon et du sein par la metformine provoque un blocage du cycle cellulaire en phase G2/M et une augmentation de l'apoptose. Dans les lignées cellulaires de cancer de la prostate, la metformine induit un arrêt du cycle cellulaire en phase G0/Gl par l'intermédiaire d'une diminution de l'expression de la cycline D1 [33] (Figure 3).

\section{Reprogrammation métabolique de la cellule cancéreuse}

Le métabolisme des cellules cancéreuses est basé essentiellement sur l'utilisation de glucose pour la production d'énergie même en présence d'oxygène. Cette caractéristique conduit les cellules cancéreuses à utiliser de manière préférentielle la glycolyse en conditions d'aérobie (effet Warburg [52] ou glycolyse aérobie) aux dépens de la phosphorylation oxydative mitochondriale. Néanmoins, les cellules cancéreuses utilisent leurs mitochondries pour la production de métabolites (malate, citrate) nécessaires aux différentes voies de biosynthèse utilisées lors de la prolifération cellulaire. 
Ainsi, en bloquant la chaîne respiratoire, la metformine va inhiber cette adaptation métabolique et provoquer une crise énergétique majeure forçant les cellules cancéreuses à s'engager dans un processus de survie (induction de la glycolyse et/ou de l'autophagie), dont l'échec aboutit à la mort cellulaire par apoptose. Par exemple, les cellules tumorales présentant une perte de fonction de p53 sont incapables de répondre aux modifications métaboliques imposées par la metformine (qui nécessitent l'activation de la protéine p53 par I'AMPK) et meurent par apoptose [36]. De même, les cellules cancéreuses déficientes en LKBI (qui présentent une faible activité AMPK) sont plus sensibles à la déplétion en ATP imposée par la metformine en raison de leur incapacité à restaurer leur balance énergétique par activation de I'AMPK. Dans ces conditions, la metformine devient alors un agent cytotoxique sélectif des cellules cancéreuses.

\section{Effet de la metformine sur le microenvironnement tumoral}

Des modifications du microenvironnement tumoral pourraient également participer aux effets bénéfiques de la metformine. II a été rapporté que la metformine stimule la génération de cellules $T$ mémoire $\mathrm{CD} 8^{+}$, permettant une meilleure protection immunitaire antitumorale [37]. Une inhibition de l'angiogenèse tumorale par la metformine a été évoquée par une réduction des taux circulants de PAI-1 (plasminogen activator inhibitor-1) et de VEGF (vascular endothelial growth factor) (Figure 3), mais des effets opposés ont été observés dans une autre étude avec une augmentation de la densité microvasculaire intratumorale des xénogreffes de cellules tumorales [31].

\section{Effet préventif de la metformine sur le développement tumoral}

La metformine participe à la réponse aux dommages à l'ADN par une activation sélective de la protéine ATM (ataxia telangiectasia mutated), mais également en bloquant la production d'espèces réactives de l'oxygène induite par la transformation par l'oncogène Ras [38]. Par ailleurs, la metformine contribue à la prévention du développement tumoral par le contrôle de l'ontogenèse des cellules souches cancéreuses en ciblant la transition épithélio-mésenchymateuse et la différenciation de ces cellules $[39,40]$. Cette action spécifique de la metformine sur les cellules souches cancéreuses a ouvert de nouvelles perspectives dans la prévention du cancer en raison de la résistance de ces cellules à la chimiothérapie. En effet, il est important de noter que le traitement par la metformine a été associé à un meilleur taux de réponse dans le cadre de chimiothérapies du cancer du sein [41]. Cet effet synergique de la metformine sur la cytotoxicité d'agents chimiothérapeutiques a été confirmé in vitro et s'est révélé efficace pour réduire la résistance à ces produits, renforçant l'idée d'une utilisation de la metformine comme adjuvant en chimiothérapie [42]. Enfin, des études récentes ont suggéré un rôle de la metformine dans l'inhibition du potentiel invasif des cellules cancéreuses et la formation de métastases par une diminution de l'activité des métalloprotéinases MMP2 et - 9 $[40,43]$ (Figure 3).

Ainsi, après plus de 50 ans au service du diabète, cette vieille molécule pourrait avoir une seconde jeunesse avec de nouvelles perspectives dans le traitement du cancer. Cependant, il reste important d'iden- tifier les patients qui pourraient bénéficier des effets bénéfiques de la metformine sur la prévention ou le traitement du cancer, en recherchant des biomarqueurs prédictifs de son effet thérapeutique. En effet, la vulnérabilité des cellules cancéreuses à la metformine dépend du type de cancer, de la présence de mutations (par exemple, pour LKB1, p53 ou des polymorphismes du gène $\mathrm{OCTl}$ ) et de l'environnement de la tumeur. Sur la base des caractéristiques pharmacocinétiques de la phenformine (perméabilité membranaire, forte inhibition du complexe I), plusieurs auteurs ont proposé que cette molécule, aujourd'hui retirée de notre arsenal thérapeutique, puisse être utilisée comme un agent antinéoplasique bien plus efficace que la metformine [34]. La phenformine présente des effets antitumoraux très puissants en conditions de stress métabolique lorsque l'axe LKBl/AMPK n'est pas fonctionnel [44]. Les travaux futurs devront chercher à déterminer les doses optimales et tolérables de metformine (et de phenformine) dans le traitement des différents types de cancer. Plus d'une centaine d'essais cliniques de phase II et III évaluant l'utilisation de la metformine en cancérologie sont d'ores et déjà en cours².

\section{Effet neuroprotecteur de la metformine, un espoir pour les maladies neurodégénératives?}

Des travaux récents ont permis d'envisager de nouvelles indications thérapeutiques pour la metformine dans le traitement de pathologies neurodégénératives. La metformine pourrait avoir un effet neuroprotecteur et prophylactique chez les patients avec une prédisposition pour la maladie d'Alzheimer [45]. L'hyperphosphorylation de la protéine tau associée aux microtubules est un élément majeur dans la pathogenèse de la maladie d'Alzheimer en provoquant les dégénérescences neurofibrillaires. La metformine provoque la déphosphorylation de la protéine tau par activation de la protéine phosphatase PP2A, ce qui limite alors la progression de la maladie. D'autres cibles d'action de la metformine sur l'intégrité du système nerveux central sont l'inhibition de la réponse inflammatoire et du stress oxydant, comme démontré dans un modèle expérimental d'encéphalomyélite allergique [46]. Un effet potentiel de la metformine sur les fonctions cognitives a également été suggéré après l'observation d'un retard dans l'apparition des troubles cognitifs dans un modèle murin de la maladie de Huntington [47]. De plus, une étude vient de révéler que la metformine favorise la neurogenèse et améliore les capacités d'apprentissage et la

${ }^{2}$ Liste consultable sur le site http://www.clinicaltrials.gov 
mémoire spatiale liées à l'hippocampe [48]. La metformine permet la phosphorylation de CBP (CREB binding protein), un coactivateur transcriptionnel portant une activité histone acétyltransférase, par la

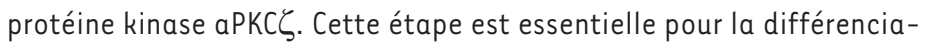
tion des précurseurs radiaires et la formation des nouveaux neurones de l'hippocampe.

\section{Effet sur la longévité : la metformine, un élixir de longue vie?}

La protection conférée par la metformine contre le diabète, les maladies cardiovasculaires et le cancer rappelle les propriétés antivieillissement de la restriction calorique (Figure 4). En effet, une restriction calorique de 25 à $60 \%$ est un moyen bien connu pour augmenter l'espérance de vie dans toutes les espèces animales. Ainsi, des propriétés mimétiques de la restriction calorique ont été décrites pour la metformine, avec un allongement de la durée de vie chez le nématode Caenorhabditis elegans ou le rongeur. Chez la souris non diabétique, la metformine mime les effets de la restriction calorique sur le profil d'expression génique et prévient l'apparition du diabète, des maladies cardiovasculaires et du cancer [49]. Les effets de la metformine chez le nématode sont dépendants de la voie $\mathrm{LKBl} / \mathrm{AMPK}$ et impliquent une augmentation des défenses anti-oxydantes [50]. Récemment, il a été suggéré que la metformine augmente indirectement la durée de vie du nématode en perturbant le métabolisme de Escherichia coli, son partenaire trophique microbien [51]. La question se pose de l'existence de mécanismes similaires chez l'homme avec une altération du métabolisme du microbiote intestinal, et de savoir si ces modifications peuvent participer aux effets thérapeutiques de la metformine.

\section{Conclusion}

La metformine est actuellement l'antidiabétique le plus couramment utilisé dans le traitement du diabète de type 2. Ce succès relève de multiples facteurs: son efficacité, sa sécurité d'emploi, sa bonne tolérance et son faible coût de production. Le foie est la principale cible d'action de la metformine où elle inhibe de manière modérée le complexe I de la chaîne respiratoire mitochondriale. Ceci provoque une diminution de la charge énergétique du foie, ce qui a pour conséquence une réduction de la production hépatique de glucose. Dans ces conditions de stress énergétique, l'activation du senseur énergétique AMPK n'a cependant pas de rôle dans l'inhibition de la gluconéogenèse par la metformine. Cette molécule offre également l'avantage de limiter l'apparition des complications cardiovasculaires liées au diabète en induisant un préconditionnement myocardique. De plus, la metformine a montré un avantage significatif dans la diminution du risque de développement tumoral par un contrôle de la différenciation et de la prolifération cellulaire. De nouvelles indications thérapeutiques pourraient encore voir le jour avec le traitement des maladies neurodégénératives. Il est donc fort à parier que cette vieille molécule n'ait pas fini de nous étonner. $\diamond$

\section{SUMMARY}

New promises for metformin: advances in the understanding of its mechanisms of action

Metformin is currently the drug of first choice for the treatment of type 2 diabetes. However, although prescribed since the end of the 1950s, the mechanism of action of metformin remains as yet incompletely understood but recent work has unveiled novel and surprising properties. Epidemiological reports have suggested that metformin protects against heart failure and has antitumor properties independent of its anti-hyperglycemic effect. Here, we review the proposed mechanisms for metformin action in diabetes, cardiovacular diseases and cancer. $\diamond$

\section{LIENS D'INTÉRÊT}

Les auteurs ne déclarent avoir aucun lien d'intérêt concernant les données publiées dans cet article.

\section{RÉFÉRENCES}

1. Bailey C, Campbell I. Metformin: the gold standard. A scientific handbook. Chichester, UK : Wiley, $2007: 288 \mathrm{p}$.

2. Sterne J. Du nouveau dans les antidiabétiques. La NN diméthylamino guanyl guanidine (NNDG). Maroc Med 1957 ; 36 : 1295-6.

3. UKPDS. Effect of intensive blood-glucose control with metformin on complications in overweight patients with type 2 diabetes (UKPDS 34) Lancet 1998 ; 352 : 854-65.

4. Stades AM, Heikens JT, Erkelens DW, et al. Metformin and lactic acidosis: cause or coincidence? A review of case reports. J Intern Med 2004 ; 255 : 179-87.

5. Lee A, Morley JE. Metformin decreases food consumption and induces weight loss in subjects with obesity with type II non-insulin-dependent diabetes. Obes Res $1998 ; 6: 47-53$.

6. Lin $\mathrm{HZ}$, Yang SQ, Chuckaree $C$, et al. Metformin reverses fatty liver disease in obese, leptin-deficient mice. Nat Med 2000 ; 6 : 998-1003.

7. Marchesini G, Brizi M, Bianchi G, et al. Metformin in non-alcoholic steatohepatitis. Lancet $2001 ; 358: 893-4$.

8. Foretz M, Viollet B. Mécanisme d'action hépatique de la metformine dans le diabète de type 2. Med Mal Metab 2009 ; $3: 48-54$.

9. Foretz M, Viollet B. Mécanisme d'inhibition de la production hépatique de glucose par la metformine. Med Sci (Paris) $2010 ; 26: 663-6$.

10. Knowler WC, Barrett-Connor $\varepsilon$, Fowler SE, et al. Reduction in the incidence of type 2 diabetes with lifestyle intervention or metformin. N Engl J Med 2002 ; $346: 393-403$.

11. Rowan JA, Hague WM, Gao W, et al. Metformin versus insulin for the treatment of gestational diabetes. N Engl J Med 2008 ; 358 : 2003-15.

12. Lord JM, Flight IH, Norman RJ. Metformin in polycystic ovary syndrome: systematic review and meta-analysis. Br Med J 2003 ; 327 : 951-3.

13. Shu Y, Sheardown SA, Brown C, et al. Effect of genetic variation in the organic cation transporter 1 (OCT1) on metformin action. J Clin Invest 2007 ; $117: 1422-31$.

14. Argaud D, Roth H, Wiernsperger N, Leverve XM. Metformin decreases gluconeogenesis by enhancing the pyruvate kinase flux in isolated rat hepatocytes. Eur J Biochem $1993 ; 213$ : 1341-8.

15. $\varepsilon l-M i r M Y$, Nogueira V, Fontaine $\varepsilon$, et al. Dimethylbiguanide inhibits cell respiration via an indirect effect targeted on the respiratory chain complex I. J Biol Chem $2000 ; 275: 223-8$.

16. Owen MR, Doran $\varepsilon$, Halestrap AP. Evidence that metformin exerts its antidiabetic effects through inhibition of complex 1 of the mitochondrial respiratory chain. Biochem J 2000 ; $348: 607-14$.

17. Logie L, Harthill J, Patel K, et al. Cellular responses to the metal-binding properties of metformin. Diabetes $2012 ; 61: 1423-33$.

18. Zhou G, Myers R, Li Y, et al. Role of AMP-activated protein kinase in mechanism of metformin action. J Clin Invest 2001 ; 108 : 1167-74.

19. Foretz M, Taleux N, Guigas $B$, et al. Régulation du métabolisme énergétique par l'AMPK. Med Sci (Paris) $2006 ; 22: 381-8$. 


\section{RÉFÉRENCES}

20. Foretz M, Viollet B. Regulation of hepatic metabolism by AMPK. J Hepatol 2011 ; $54: 827-9$.

21. Shaw RJ, Lamia KA, Vasquez D, et al. The kinase LKBI mediates glucose homeostasis in liver and therapeutic effects of metformin. Science $2005 ; 310: 1642-6$.

22. Foretz $M$, Hebrard S, Leclerc J, et al. Metformin inhibits hepatic gluconeogenesis in mice independently of the LKBI/AMPK pathway via a decrease in hepatic energy state. J Clin Invest $2010 ; 120: 2355-69$

23. Miller RA, Chu $Q$, Xie J, et al. Biguanides suppress hepatic glucagon signalling by decreasing production of cyclic AMP. Nature $2013 ; 494: 256-60$.

24. Yin M, van der Horst IC, van Melle JP, et al. Metformin improves cardiac function in a nondiabetic rat model of post-MI heart failure. Am J Physiol Heart Circ Physiol 2011 ; 301 : H459-68.

25. Gundewar S, Calvert JW, Jha S, et al. Activation of AMP-activated protein kinase by metformin improves left ventricular function and survival in heart failure. Circ Res $2009 ; 104: 403-11$.

26. MacDonald MR, Eurich DT, Majumdar SR, et al. Treatment of type 2 diabetes and outcomes in patients with heart failure: a nested case-control study from the U.K. General practice research database. Diabetes care $2010 ; 33: 1213-8$.

27. Xie Z, Lau K, Eby B, et al. Improvement of cardiac functions by chronic metformin treatment is associated with enhanced cardiac autophagy in diabetic OVE26 mice. Diabetes $2011 ; 60: 1770-8$

28. Evans JM, Donnelly LA, Emslie-Smith AM, et al. Metformin and reduced risk of cancer in diabetic patients. Br Med J $2005 ; 330$ : 1304-5.

29. Beck $\varepsilon$, Scheen AJ. Quels bénéfices antitumoraux attendre de la metformine? Ann Endocrinol (Paris) $2013 ; 74: 137-47$.

30. Memmott RM, Mercado JR, Maier CR, et al. Metformin prevents tobacco carcinogen-induced lung tumorigenesis. Cancer Prev Res $2010 ; 3: 1066-76$.

31. Viollet B, Foretz M. Metformine et cancer. Du diabète au cancer : de nouvelles perspectives thérapeutiques pour la metformine. Med Mal Metab 2011 ; 5 : 29-37.

32. Zakikhani M, Dowling R, Fantus IG, et al. Metformin is an AMP kinase-dependent growth inhibitor for breast cancer cells. Cancer Res $2006 ; 66: 10269-73$.

33. Ben Sahra I, Laurent $K$, Loubat A, et al. The antidiabetic drug metformin exerts an antitumoral effect in vitro and in vivo through a decrease of cyclin Dl level. Oncogene $2008 ; 27: 3576-86$.

34. Huang $X$, Wullschleger $S$, Shpiro N, et al. Important role of the LKBI-AMPK pathway in suppressing tumorigenesis in PTEN-deficient mice. Biochem J 2008 ; 412 : 211-21.

35. Ben Sahra I, Regazzetti C, Robert G, et al. Metformin, independent of AMPK, induces mTOR inhibition and cell-cycle arrest through REDDl. Cancer Res $2011 ; 71: 4366-72$.

36. Buzzai M, Jones RG, Amaravadi RK, et al. Systemic treatment with the antidiabetic drug metformin selectively impairs p53-deficient tumor cell growth. Cancer Res $2007 ; 67: 6745-52$.

37. Pearce EL, Walsh MC, Cejas PJ, et al. Enhancing CD8 T-cell memory by modulating fatty acid metabolism. Nature $2009 ; 460: 103-7$.

38. Algire C, Moiseeva 0 , Deschenes-Simard X, et al. Metformin reduces endogenous reactive oxygen species and associated DNA damage. Cancer Prev Res $2012 ; 5$ : 536-43.

39. Del Barco S, Vazquez-Martin A, Cufi S, et al. Metformin: multi-faceted protection against cancer. Oncotarget $2011 ; 2: 896-917$.

40. Cerezo M, Tichet $M$, Abbe $P$, et al. Metformin blocks melanoma invasion and metastasis development in a AMPK/p53-dependent manner. Mol Cancer Ther 2013 ; 12 : 1605-15.
41. Jiralerspong S, Palla SL, Giordano SH, et al. Metformin and pathologic complete responses to neoadjuvant chemotherapy in diabetic patients with breast cancer. J Clin Oncol $2009 ; 27$ : 3297-302.

42. Hirsch HA, Iliopoulos D, Tsichlis PN, Struhl K. Metformin selectively targets cancer stem cells, and acts together with chemotherapy to block tumor growth and prolong remission. Cancer Res $2009 ; 69$ : 7507-11.

43. Hwang $Y P$, Jeong HG. Metformin blocks migration and invasion of tumour cells by inhibition of matrix metalloproteinase -9 activation through a calcium and protein kinase Calpha-dependent pathway: phorbol-12myristate-13-acetate-induced/extracellular signal-regulated kinase/ activator protein-1. BrJ Pharmacol $2010 ; 160: 1195-211$

44. Shackelford DB, Abt $\varepsilon$, Gerken $L$, et al. $L K B 1$ inactivation dictates therapeutic response of non-small cell lung cancer to the metabolism drug phenformin. Cancer Cell 2013; $23: 143-58$.

45. Kickstein $\varepsilon$, Krauss $S$, Thornhill $P$, et al. Biguanide metformin acts on tau phosphorylation via $\mathrm{mTOR} /$ protein phosphatase $2 \mathrm{~A}$ (PP2A) signaling. Proc Natl Acad Sci USA 2010 ; 107 : 21830-5

46. Paintlia AS, Paintlia MK, Mohan S, et al. AMP-activated protein kinase signaling protects oligodendrocytes that restore central nervous system functions in an experimental autoimmune encephalomyelitis model. Am J Pathol 2013 ; 183 : 526-41.

47. Ma TC, Buescher JL, Oatis B, et al. Metformin therapy in a transgenic mouse model of Huntington's disease. Neurosci Lett 2007 ; 411 : 98-103.

48. Wang J, Gallagher D, DeVito LM, et al. Metformin activates an atypical PKC-CBP pathway to promote neurogenesis and enhance spatial memory formation. Cell Stem Cell $2012 ; 11: 23-35$

49. Anisimov VN, Berstein LM, Popovich IG, et al. If started early in life, metformin treatment increases life span and postpones tumors in female SHR mice. Aging (Albany NY) $2011 ; 3$ : 148-57.

50. Onken B, Driscoll M. Metformin induces a dietary restriction-like state and the oxidative stress response to extend $C$. elegans Healthspan via AMPK, LKBI, and SKN-1. PLoS One 2010 ; 5 : e8758.

51. Cabreiro F, Au C, Leung KY, et al. Metformin retards aging in C. elegans by altering microbial folate and methionine metabolism. Cell $2013 ; 153: 228$ 39.

52. Razungles J, Jalaguier S, Cavaillès V, Teyssier C. L'effet Warburg : de la théorie du cancer aux applications thérapeutiques en cancérologie. Med Sci (Paris) $2013 ; 29: 1026-33$

53. Julien LA, Roux PP. mTOR, the mammalian target of rapamycin. Med Sci (Paris) $2010 ; 26: 1056-60$
TIRÉS À PART

M. Foretz et B. Viollet

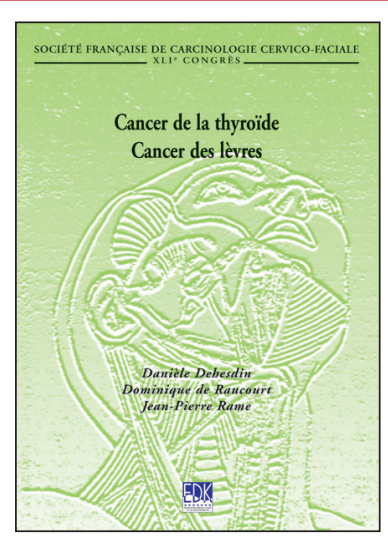

ISBN : 978-2-8425-4137-8 264 pages
Bon de commande à retourner à EDK, 25, rue Daviel - 75013 Paris

Tél. : 0158101905 - Fax : 0143293262 - E-mail : edk@edk.fr

NOM :

Prénom :

Adresse :

Code postal :

Ville :

Pays :

Fonction :

Je souhaite recevoir l'ouvrage Cancer de la thyroïde - Cancers des lèvres : $35 €+3 €$ de port $=\mathbf{3 8} €$ TTC

en ................. exemplaire, soit un total de ..................................... €

$\square$ Par chèque, à l'ordre de $\mathbf{E} \mathbf{D} \mathbf{K}$

$\square$ Par carte bancaire : $\square$ Visa $\square$ Eurocard/Mastercard

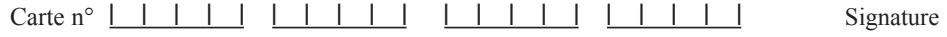

Date d'expiration: $\quad \underline{1 \mid} \underline{1 \mid}$

$\mathrm{N}^{\circ}$ de contrôle au dos de la carte : $\quad$ । $\mid$ 